\title{
2019 Pediatric Initiative Network: Progress, Proceedings, and Plans
}

\author{
Leena Nahata, MD, ${ }^{1-3}$ Veronica Gomez-Lobo, MD, ${ }^{4}$ Lillian Meacham, MD, ${ }^{5,6}$ Leslie Appiah, MD, \\ Krista Childress, MD, ${ }^{9,10}$ Holly Hoefgen, MD, ${ }^{11}$ Maggie Dwiggins, MD, ${ }^{12}$ Stacy Whiteside, APRN, ${ }^{13}$ \\ Kari Bjornard, MD, MPH, ${ }^{14,15}$ Julie Rios, MD, ${ }^{16,17}$ Antoinette Anazodo, MD, PhD, ${ }^{18,19}$ \\ Courtney Finlayson, MD,20,21 Olivia Frias, MSN, BSN, ${ }^{17}$ \\ Teresa Woodruff, $\mathrm{PhD}^{22}$ and Molly Moravek, MD, $\mathrm{MPH}^{23}$
}

Impairment of fertility and sexual/reproductive health are common after oncologic therapy, and are known to have negative impacts on romantic relationships and psychosocial well-being among childhood cancer survivors. The Pediatric Initiative Network (PIN) is an international, multidisciplinary group of providers within the Oncofertility Consortium dedicated to preserving and protecting the fertility of children and adolescents at risk for infertility due to medical conditions or treatments. The PIN and its Best Practices and Research committees meet virtually throughout the year, with one annual in-person meeting. The purpose of this "proceedings" is to highlight key discussion points from the annual PIN meeting which took place on November 11, 2019, to 1) provide a context for pediatric groups across the country on what oncofertility programs are currently doing and why, and 2) inform stakeholders of past, present and future initiatives that may be of value to them and the patient populations they serve.

Keywords: oncofertility, childhood cancer, fertility preservation

\section{Introduction}

$\mathbf{N}$ EARLY 300,000 INDIVIDUALS UNDER the age of 20 are diagnosed with cancer around the world each year. ${ }^{1}$ Treatment advances have led to increasing survival rates, highlighting the importance of addressing and mitigating late effects of oncologic therapies. ${ }^{2,3}$ For example, impairment of fertility and sexual/reproductive health often occur due to surgery, chemotherapy, and radiation and are known to have negative impacts on future romantic relationships and psychosocial well-being. $^{3-9}$ These are uniquely challenging topics to consider in the pediatric and adolescent age group,

${ }^{1}$ Center for Biobehavioral Health, Abigail Wexner Research Institute at Nationwide Children's Hospital, Columbus, Ohio, USA.

${ }^{2}$ Division of Endocrinology, Nationwide Children's Hospital, Columbus, Ohio, USA.

${ }^{3}$ Department of Pediatrics, The Ohio State University College of Medicine, Columbus, Ohio, USA.

${ }^{4}$ Division of Pediatric and Adolescent Gynecology, Eunice Kennedy Shriver National Institute of Child Health and Human Development, Bethesda, Maryland, USA.

${ }^{5}$ Aflac Cancer Center, Children's Healthcare of Atlanta, Atlanta, Georgia, USA.

${ }^{6}$ Division of Hematology/Oncology and Endocrinology, Department of Pediatrics, Emory University, Atlanta, Georgia, USA.

${ }^{7}$ Department of Obstetrics and Gynecology, The University of Colorado School of Medicine Anschutz Medical Campus, Denver, Colorado, USA.

${ }^{8}$ Children's Hospital Colorado, Denver, Colorado, USA.

${ }^{9}$ Division of Gynecologic Specialties, Department of Gynecology and Obstetrics, Emory University, Atlanta, Georgia, USA.

${ }^{10}$ Division of Pediatric Surgery, Children's Healthcare of Atlanta, Atlanta, Georgia, USA.

${ }^{11}$ Department of Obstetrics and Gynecology, Division of Pediatric and Adolescent Gynecology, Washington University School of Medicine, St. Louis, Missouri, USA.

${ }^{12}$ Medstar Washington Hospital Center, Washington, District of Columbia, USA.

${ }^{13}$ Division of Hematology/Oncology, Nationwide Children's Hospital, Columbus, Ohio, USA.

Departments of ${ }^{14}$ Epidemiology and Cancer Control and ${ }^{15}$ Oncology, St. Jude Children's Research Hospital, Memphis, Tennessee, USA.

${ }^{16}$ Department of Obstetrics and Gynecology, University of Cincinnati, Cincinnati, Ohio, USA.

${ }^{17}$ Comprehensive Fertility Care and Preservation Program, Division of Pediatric and Adolescent Gynecology, Cincinnati Children's Hospital Medical Center, Cincinnati, Ohio, USA.

${ }^{10}$ Kids Cancer Centre, Sydney Children's Hospital, Sydney, Australia.

${ }^{19}$ Nelune Comprehensive Cancer Centre, Prince of Wales Hospital, Sydney, Australia.

${ }^{20}$ Division of Pediatric Endocrinology, Ann \& Robert H. Lurie Children's Hospital of Chicago, Chicago, Illinois, USA.

Departments of ${ }^{21}$ Pediatrics and ${ }^{22}$ Obstetrics and Gynecology, Northwestern University Feinberg School of Medicine, Chicago, Illinois, USA.

${ }^{23}$ Division of Reproductive Endocrinology and Infertility, Department of Obstetrics and Gynecology, University of Michigan, Ann Arbor, Michigan, USA. 
as (1) established fertility preservation (FP) technologies have not historically been available for prepubertal youth; (2) developmental considerations may limit capacity for futureoriented planning, including formation of parenthood goals and FP decisions; (3) shared decision making models are needed to facilitate discussions between providers, parents, and patients; and (4) ethical and legal challenges often arise during discussions about these topics among minors. To optimize long-term psychosocial and reproductive outcomes, there is a critical need for providers from different disciplines and regions to collaborate to establish evidence-based "best" oncofertility practices for pediatric and adolescent populations.

The Pediatric Initiative Network (PIN) is an international group of providers within the Oncofertility Consortium dedicated to preserving and protecting the fertility of children and adolescents at risk for infertility due to medical conditions or treatments. ${ }^{10-12}$ In a span of 6 years, the PIN has established itself as an integral and necessary component of the Oncofertility Professional Engagement Network (OPEN) of the Oncofertility Consortium and a strengthening voice within the field. ${ }^{13}$ The group has grown rapidly, with 102 active members from 62 institutions, including physicians, researchers, advanced practitioners, nurses, psychologists, social workers, patient navigators, and trainees from each of these groups. Numerous medical disciplines are represented, including oncology, endocrinology, pediatric gynecology, reproductive endocrinology, and urology. Two major committees have been established within the PIN-Best Practices and Research. There are no dues or requirements to join the PIN; individuals can email the PIN Chair and committee chairs, explaining their involvement in oncofertility and interest in joining: http://oncofertility.northwestern.edu/ pediatric-initiative-network. The PIN and its committees/ subcommittees meet virtually (through WebEx/conference calls/emails/listservs) throughout the year and with one annual in-person meeting. The purpose of this "proceedings" is to highlight key discussion points from the annual PIN meeting, which took place on November 11, 2019, in Chicago, IL, to 1) provide a context for pediatric groups across the country on what oncofertility programs are currently doing and why, and 2) inform stakeholders of past, present, and future initiatives that may be of value to them and the patient populations they serve.

\section{Oncofertility Program Development: Successes and Ongoing Challenges}

A total of 103 people from 60+ institutions in 10 countries participated in the 2019 PIN meeting. The first main agenda item was to discuss oncofertility program development, including the following key questions: (1) which institutions have a formal FP/reproductive health program; (2) which programs have a dedicated patient navigator/coordinator; (3) are reproductive endocrinologists and urologists available to perform FP procedures as needed; (4) which strategies have been implemented to maximize access; (5) what are ongoing barriers; (6) are ovarian tissue cryopreservation (OTC) and testicular tissue cryopreservation (TTC) being offered and to which patient populations; and (7) how are costs being handled for cryopreservation, processing, and storage?

Of the represented institutions, 31 reported having a formal FP program, and 13 of those had a dedicated patient navi- gator/coordinator. In the United States, OTC is currently offered at 16 of these institutions, and TTC is offered at 12. Representatives from other countries reported their experiences as well; for example, attendees from Japan reported that there are over 42 centers offering tissue cryopreservation in their country. All of the institutions offering tissue cryopreservation included gonadotoxic cancer therapy and bone marrow transplant for nononcologic conditions as indications, but only select institutions were including patients with differences of sex development, rheumatologic disorders requiring high dose cyclophosphamide, Turner Syndrome, genetic predispositions to primary ovarian insufficiency, or transgender patients. Financial considerations were frequently noted to be a concern, with several of the institutions offering tissue processing for OTC at no charge to the patient (e.g., using research funds since it has historically been considered experimental), and others offer processing for $\$ 400-1000$. The majority of programs reported surgical costs for OTC ranging from $\$ 5000$ to $\$ 8000$; those billing insurance reported that coverage had been inconsistent and unpredictable. Access to established FP procedures (e.g., oocyte cryopreservation and testicular sperm extraction) was not universal. Of the represented institutions, $20 \mathrm{had}$ access to a reproductive endocrinologist if needed for pediatric and adolescent FP, and 19 had a readily available reproductive urologist.

Attendees with established FP programs at their institutions also discussed ways to maximize patient access to FP services. Four programs described an "opt-out" fertility consult embedded in the oncology order set, ${ }^{14} 2$ programs used a "hard stop" to inquire about FP in the electronic medical record (EMR), and several others described dedicated inpatient/outpatient order sets for FP. Participants from several other institutions reported receiving patient lists of upcoming patients, either for tumor board meetings or for state reporting purposes. Ease of referral also improved access, including having a patient navigator/coordinator and having a dedicated email address including the entire FP team. Education of floor nurses and house officers was also identified as a strategy to increase referrals, with some programs incorporating information about FP into hospital orientation. Finally, one program reported implementing a policy that floor nurses can place the order for the FP consult (similar to how a nurse can request a social work consult), which was anticipated to increase inpatient consults.

Several barriers to program implementation and success were also identified. Even among those institutions with established FP programs, all but one institution felt their program could be improved. One of the biggest barriers identified was related to funding - both for program building and to assist with patient costs. Ongoing difficulties securing philanthropy were discussed, and several who had secured philanthropic funds to assist patients noted concerns about this approach potentially being viewed as "enticement" or "coercion" to pursue FP by institutional ethics boards and review boards. Participants also discussed difficulty securing institutional support for protected time for providers to dedicate to a FP program or to hire a patient navigator/coordinator. Potential solutions offered by established programs included having a navigator bill for consult services to help cover their salary (and considering credentials needed to bill, for example, advanced practice nurse vs. registered nurse) 
and emphasizing to institution leadership that a formal FP program is necessary or contributes to national benchmarks such as U.S. News and World Report rankings (in disciplines such as Oncology and Urology). Nursing research or specialty roles are also necessary for nursing magnet status and can be fulfilled by nurses serving as a patient navigator/coordinator or research coordinator.

The lack of standardization or an accreditation process across programs in the United States was also identified as a barrier to programmatic success. It was suggested that quality indicators be developed for pediatric FP programs, similar to the system currently in place across Australian programs. Regarding tissue cryopreservation, programs have found that slow freezers with which to freeze the tissue are becoming more scarce, as oocyte and sperm cryopreservation have moved to vitrification protocols. Some programs have moved to vitrification of tissue, while others noted the ability to buy used slow freezers from other programs; there has also been a trend toward using a centralized tissue cryopreservation center. Finally, difficulty obtaining institutional review board (IRB) approval was also noted to be a barrier to tissue cryopreservation at some institutions, because even when OTC was considered experimental it wasn't considered "research" by some IRBs (in the absence of a clear research question) and TTC has often been considered "too experimental." Suggestions from institutions who had secured IRB approval included preemptive discussions with IRB representatives before submission or attending the IRB meeting where the topic is being discussed; FP programs with clinical research coordinators may be able to navigate these processes more efficiently. Some programs are pairing a research study with OTC, such as creating a database or surveying patients on their experience.

\section{Best Practices Committee}

The mission of the Best Practices Committee is to develop strategies to optimize fertility related care for at-risk youth. At the annual Oncofertility Consortium meeting, the committee identifies initiatives important to the PIN and develops projects that can be accomplished during the subsequent 12 months. During 2019, the Best Practices Committee implemented three initiatives, which were presented at the meeting: (1) An Infertility Risk Stratification System; (2) Development of an Oncofertility Patient Navigation Committee; and (3) A single journal series of review articles dedicated to Pediatric, Adolescent and Young Adult (AYA) FP and Reproductive Health in Survivorship.

\section{Risk stratification}

The risk stratification project began because of the discrepancies in cut points used to classify level of risk for future infertility after childhood cancer therapy. The discrepancies made comparisons across research studies challenging and led to variability in clinical practice at different sites. A working group of 27 PIN members reviewed current methods in use clinically and in research to stratify risk for infertility, performed a literature review, ${ }^{4,15-29}$ and then developed risk stratification grids. Separate stratification grids were developed for female and male patients categorizing risk for future infertility/gonadal failure into minimally increased risk, significantly increased risk, and a high level of increased risk after exposure to gonadotoxic cancer therapies. The exposures known to place survivors at risk for infertility/gonadal failure were identified from the Children's Oncology Group Long-Term Follow-Up Guidelines for Survivors of Pediatric, Adolescent, and Young Adult Cancers and include: alkylating and heavy metal chemotherapy, radiation to the gonad or hypothalamus, hematopoietic stem cell transplant, and surgery on the reproductive tract. ${ }^{15}$ These grids will soon be published ${ }^{30}$ to serve as a resource for clinicians and researchers.

\section{Oncofertility Patient Navigator Subcommittee}

During 2019, the Oncofertility Patient Navigator Subcommittee was established after a national survey of PIN members demonstrated the essential role of navigators in providing timely and comprehensive fertility and reproductive health care to youth and families before, during, and after cancer treatment. ${ }^{11}$ This group has expanded rapidly and now includes 25 participants from 23 institutions across the United States and Australia. Initial efforts to establish this occurred at the 2018 Oncofertility Consortium meeting among attendees; the subcommittee has expanded over 2019 strictly by word of mouth. Strategies to further expand membership within the United States and internationally are underway for the upcoming year. The group has participated in bimonthly conference calls throughout the year with three main goals: to provide a forum to address the unique needs of fertility navigators; to create a network of cooperative programs in all stages of development; and to develop navigator specific projects and contributions to the Oncofertility Consortium. In early 2019 , members initiated a pilot fertility program survey sent to existing subcommittee members. This work will continue and expand over the next year with a plan to disseminate to navigators across the Consortium. The survey will provide program and navigator information for benchmarking, establish a network for collaboration, and facilitate access to mentorship for programs during their early stages. In addition, the Navigator subcommittee coordinated a working lunch panel presentation during the 2019 Oncofertility Consortium Conference entitled "Fertility Preservation Legislation and Loopholes." The panel focused on FP insurance mandates, practical approaches to advocacy, and descriptions of navigator-initiated legislative efforts seeking funding for FP and reproductive health.

\section{Reproductive health in survivorship}

A final project from the 2019 Best Practices Committee consists of a series of articles focusing on Reproductive Health in Pediatric and AYA cancer patients, with the plan for them to be published as a special edition series. The eight articles will include: Pediatric Reproductive Tumors, Male Pediatric and AYA Reproductive Survivorship, Female Pediatric and AYA Reproductive Survivorship, Reproductive Late Effects after BMT, Fertility Considerations in AYA Survivorship, Psychosexual Functioning in Survivorship, Reproductive Health Literacy, and Reproductive Care Navigation in Survivorship. The purpose of this effort is to collate reproductive concerns in cancer survivorship in one easily referenceable location. Critical gaps discussed in these reviews include diagnosis and management of diminished ovarian reserve, identification and treatment of genital graft 
versus host disease, screening and treatment interventions for sexual dysfunction, reproductive cancer surveillance, and hormone replacement in the setting of comorbidities. The articles will serve as the basis of future prospective research to address these critical gaps in care.

\section{Future Best Practices committee initiatives}

After soliciting ideas from the PIN membership and ascertaining the interest of members to participate in working groups, the 2020 Best Practices initiative will evaluate the use of Anti-Mullerian hormone (AMH) in survivors of childhood cancer. AMH has become the best currently available marker of ovarian reserve and has aided in counseling patients about their reproductive life span, as well as the effects of gonadotoxic therapy during cancer treatment. Several studies have reported the use of $\mathrm{AMH}$ in evaluating ovarian reserve in pediatric cancer patients who have undergone gonadotoxic therapy. ${ }^{31-35}$ However, while these studies collectively suggest that $\mathrm{AMH}$ is a sensitive marker for damage to the ovarian reserve, there are currently little data regarding how and when to use $\mathrm{AMH}$ as a screening tool in the survivorship population. In fact, current surveillance recommendations in female childhood cancer survivors from the International Late Effects of Childhood Cancer Guideline Harmonization Group do not recommend using AMH as a primary surveillance modality for primary ovarian insufficiency. ${ }^{16}$ Much of the reasoning for not recommending $\mathrm{AMH}$ as a standard method of surveillance is due to the lack of literature on $\mathrm{AMH}$ in this population. Nevertheless, AMH has been a helpful addition in the counseling and identification of patients who may have a narrowed window of reproductive potential secondary to their therapy, and further understanding of how $\mathrm{AMH}$ is used in the survivorship population may provide a benefit to future patients and providers.

In a breakout session, the working groups convened and identified project leaders, defined the goals of the project, and began to create next steps and timelines. This project will begin with a review of the current evidence regarding $\mathrm{AMH}$ surveillance in pediatric oncology survivors through a systematic review. Second, oncofertility practitioners in the PIN will be surveyed to evaluate current practices regarding AMH screening in female childhood cancer survivors. This will include questions surrounding who $\mathrm{AMH}$ testing is ordered on, at which timepoint(s) in the survivorship continuum, and when to refer these patients to a Reproductive Endocrinology and Infertility specialist. Analyzing and disseminating these data will serve as an important initial step to establishing best practices for the use of AMH in the survivorship population.

\section{Research Committee}

The mission of the PIN Research Committee is to design and implement collaborative multisite research studies to advance fertility related care for at-risk youth. As more institutions implement FP programs, many questions remain to be studied such as: patients' and families' understanding of and decision-making around FP options (both established and experimental), risks of immediate or delayed gonadal failure of older and newer therapeutic agents, long-term effects of FP procedures, as well as the ascertainment of ovarian reserve in children and adolescents. The ability to share information across sites for the purpose of research is essential, allowing a broad and expansive population base to answer these important questions. Given the increasing use of OTC and the relevance of this technology in pediatric populations, creation of a centralized OTC database was the main focus of the research committee in 2019.

\section{OTC database: lessons learned and future directions}

Although databases have been established in other countries, data and literature regarding OTC research questions remain scarce, especially regarding children and adolescents. In addition, there are regional and national differences in OTC indications and practice. The PIN's initial endeavor in U.S. OTC database creation resulted in the discovery that individual U.S. sites are both interested and incentivized to participate in a shared centralized database to share ideas and obtain IRB research approval. To date, four U.S. sites have obtained IRB approval to collect patient data in a centralized database, and three sites have entered anonymous patient information as part of their IRB approved OTC protocols. Several institutions note that the addition of the centralized database was necessary to fulfill the research requirement of their local IRB and obtain full approval for OTC protocols.

Moreover, we discovered that conducting a successful multisite database requires research support from a central data manager. Although many sites were able to obtain IRB approval and have internally funded research coordinators enter data, it became clear that a central coordinator is essential for "managing the database" and assuring data integrity. This central coordinator facilitates sharing of protocols, coordinates data-use agreements between distinct legal departments, and coordinates access to the database for each site. This coordinator can also manage transfer of deidentified data from existing databases into the centralized location, as well as send ongoing reminders to continue populating data.

Based on this prior experience, as well as that of the Australasian Oncofertility registry, ${ }^{36}$ FertiPROTEKT registry, ${ }^{37}$ and Japanese FP registry, ${ }^{38}$ in an effort to create a central repository for U.S. data regarding patients who have undergone OTC, the PIN Research Committee has decided to proceed with an effort to create a central repository for data regarding patients who have undergone OTC by: (1) revising all OTC data collection sheets into an updated centralized database to maximize information collected regarding the specifics of treatment, surgery, endocrine function, and obstetrical outcomes; (2) transitioning the database to a centralized site where there is institutional support for this endeavor with a research coordinator, database manager, designated legal team, and local grant funding for the longevity of the program; and (3) promoting vigorous academic output by encouraging each participating site to take ownership and submit research ideas, commit to participating in the chosen research idea, and have equal opportunity for authorship on every publication resulting from the database.

\section{Future research projects}

Discussion during the 2019 meeting also revealed interest in other research ideas and broader data collection regarding FP in general:

(1) Creating a TTC database: This project can be facilitated by previous experience with the OTC database. 
Using the same site and resources would allow a faster production of the database, and the fact that to date all TTC protocols are part of an ongoing single network will allow easier uptake of such a protocol and data collection at current participating sites.

(2) Optimizing basic tissue research: Although OTC is no longer considered experimental, questions remain regarding best practices for cryopreservation (e.g., slow freezing vs. vitrification) and retransplantation versus in vitro maturation of follicles, particularly from tissue obtained from prepubertal girls. Collection of tissue for basic research will improve knowledge about handling ovarian tissue for FP and future use of the tissue, which will undoubtedly improve outcomes for patients who choose OTC as a FP method. Data regarding who is doing tissue research and who is collecting tissue for research are important to enable sites to link together for future collaboration. Given the relatively small number of sites collecting tissue and actually performing research on this tissue, creating such a list was fairly simple. The decision was made that this database would be maintained within the PIN Research Committee and updated yearly. Furthermore, the committee realized that there is a need to optimize the utilization of stored tissue. Current members of the PIN intend to apply for $\mathrm{NIH}$ funding to explore best ways to maximize tissue use including but not limited to:

(i) Creating a digital library of current slide book to be shared among investigators.

(ii) Evaluating the best ways to process existing block tissues to maximize use.

(iii) Creating a system for investigators to submit proposals and for a team to review, score, and decide on which studies merit the ability to use precious tissues such as these.

(3) FP Decision-Making: Researchers within the PIN have defined study questions around FP decisionmaking (e.g., predictors of OTC/TTC uptake, factors impacting fertility testing after treatment) and would like to collaborate with sites that have FP programs. A more detailed future database may also allow direct access to sites and patients in the future.

(4) General FP Database: This database would include data from all existing FP programs throughout the PIN. Several sites are collecting detailed data regarding their fertility program. Given EMR capabilities and support at a single site, in the future it may be possible to collect detailed data from the EMR. As such, the first step would be to create a "data dictionary" to assure that all sites are collecting the same data. Furthermore, international members will share their experience and data points from their national databases. A subcommittee plans to spend the next year refining a possible future data collection dictionary for PIN participants.

\section{Conclusions}

The PIN has steadily expanded from a small network of dedicated individuals to an international multidisciplinary platform for research collaboration and integration of best practices in pediatric oncofertility. This growth reflects the increasing importance of pediatric oncofertility, as threefourths of childhood cancer survivors will experience at least one late effect of cancer treatment. Despite the potentially life-threatening sequelae of cardiac, pulmonary, and renal toxicity, reproductive late effects remain one of the most reported quality of life disruptors for survivors. ${ }^{3,39}$ Significant advancements have been made in mitigating reproductive late effects of gonadal insufficiency, infertility, genital graft-versus-host disease, sexual dysfunction, and radiation effects to the pelvic organs. ${ }^{22,40}$ Innovations in the application of assisted reproductive technologies (ART) to patients with cancer have decreased time to ART, improved safety of ART in patients with hormone sensitive tumors, and provided options for patients for whom ART are not feasible. Successful application of newly established FP therapies to the pediatric and adolescent population will require focused investigation in current areas of challenge such as familycentered FP decision-making, the applicability of AMH testing, feasibility of oocyte cryopreservation in the immediate postpubertal patient, efficiency of transplanted ovarian and testicular tissue, early identification and evidenced-based treatment of gynecologic and urologic late effects, and methods of effective data collection to more rapidly investigate and apply knowledge. Key Performance Indicators, competency frameworks, and effective communication strategies are also essential to optimal outcomes and will require dedicated effort.

The PIN harnesses the knowledge, curiosity, and desire for innovation of interdisciplinary experts and newcomers to the field. Progress requires medical providers, mental health specialists, basic scientists, stakeholders, economists, ethicists, and legal scholars. OPEN established a setting for convening these specialists through which the PIN was born and flourished to be an integral sector. PIN members work through the network to improve options and access not only for survivors of childhood cancer but also other pediatric medical conditions with impaired fertility potential. Quality of life in pediatric populations is dependent on the ability of our field to anticipate, innovate, implement, and critically evaluate to improve the care provided to patients. This collaborative power uniquely positions the PIN to meet the challenge. Future endeavors will focus on addressing the most pressing of challenges while expanding the reach and scope of the PIN.

\section{Acknowledgment}

The authors thank Lauren Ataman for her support of the PIN and assistance with these proceedings.

\section{Author Disclosure Statement}

The authors have no conflicts of interest to disclose.

\section{Funding Information}

Dr. Leena Nahata is funded by the National Institutes of Health-National Cancer Institute K08CA237338. Funding for the conference was supported by the Oncofertility Consortium Annual Conference, which is funded by the National Institutes of Health R13HD063248.

\section{References}

1. Steliarova-Foucher E, Colombet M, Ries LA, et al. International incidence of childhood cancer, 2001-10: a 
population-based registry study. Lancet Oncol. 2017;18(6): 719-31.

2. Phillips SM, Padgett LS, Leisenring WM, et al. Survivors of childhood cancer in the United States: prevalence and burden of morbidity. Cancer Epidemiol Biomarkers Prev. 2015;24(4):653-63.

3. Hudson MM, Ness KK, Gurney JG, et al. Clinical ascertainment of health outcomes among adults treated for childhood cancer. JAMA. 2013;309(22):2371-81.

4. Green DM, Liu W, Kutteh WH, et al. Cumulative alkylating agent exposure and semen parameters in adult survivors of childhood cancer: a report from the St Jude Lifetime Cohort Study. Lancet Oncol. 2014;15(11): 1215-23.

5. Armuand GM, Wettergren L, Rodriguez-Wallberg KA, Lampic C. Desire for children, difficulties achieving a pregnancy, and infertility distress 3 to 7 years after cancer diagnosis. Support Care Cancer. 2014;22(10):2805-12.

6. Lehmann VNL, Ferrante AC, Hansen-Moore JA, et al. Fertility-related perceptions and impact on romantic relationships among adult survivors of childhood cancer. J Adolesc Young Adult Oncol. 2018;7(4):409-414.

7. Woodruff TK. Oncofertility: a grand collaboration between reproductive medicine and oncology. Reproduction. 2015; 150(3):S1-10.

8. Woodruff TK. The Oncofertility Consortium-addressing fertility in young people with cancer. Nat Rev Clin Oncol. 2010;7(8):466.

9. Jeruss JS, Woodruff TK. Preservation of fertility in patients with cancer. N Engl J Med. 2009;360(9):902-11.

10. Smith BM, Duncan FE, Ataman L, et al. The National Physicians Cooperative: transforming fertility management in the cancer setting and beyond. Future Oncol. 2018; 14(29):3059-72.

11. Moravek MB, Appiah LC, Anazodo A, et al. Development of a pediatric fertility preservation program: a report from the Pediatric Initiative Network of the oncofertility consortium. J Adolesc Health. 2019;64(5):563-73.

12. Ataman LM, Rodrigues JK, Marinho RM, et al. Creating a global community of practice for oncofertility. J Glol Oncol. 2016;2(2):83-96.

13. Salama M, Ataman-Millhouse L, Sobral F, et al. Barriers and opportunities of oncofertility practice in nine developing countries and the emerging Oncofertility Professional Engagement Network. J Glob Oncol. 2018;4:1-7.

14. Saraf AJ, Stanek J, Audino A, et al. Examining predictors and outcomes of fertility consults among children, adolescents, and young adults with cancer. Pediatr Blood Cancer. 2018;65(12):e27409.

15. Children's Oncology Group. Long-term follow-up guidelines for survivors of pedaitric, Adolescent and Young Adult Cancers. 2018. Accessed November 4, 2019 from: www.survivorshipguidelines.org

16. van Dorp W, Mulder RL, Kremer LC, et al. Recommendations for premature ovarian insufficiency surveillance for female survivors of childhood, adolescent, and young adult cancer: a report from the International Late Effects of Childhood Cancer Guideline Harmonization Group in collaboration with the PanCareSurFup Consortium. J Clin Oncol. 2016;34(28):3440-50.

17. Skinner R, Mulder RL, Kremer LC, et al. Recommendations for gonadotoxicity surveillance in male childhood, adolescent, and young adult cancer survivors: a report from the International Late Effects of Childhood Cancer
Guideline Harmonization Group in collaboration with the PanCareSurFup Consortium. Lancet Oncol. 2017;18(2): e75-90.

18. Wasilewski-Masker K, Seidel KD, Leisenring W, et al. Male infertility in long-term survivors of pediatric cancer: a report from the childhood cancer survivor study. J Cancer Surviv. 2014;8(3):437-47.

19. Barton SE, Najita JS, Ginsburg ES, et al. Infertility, infertility treatment, and achievement of pregnancy in female survivors of childhood cancer: a report from the Childhood Cancer Survivor Study cohort. Lancet Oncol. 2013;14(9): 873-81.

20. Green DM, Zhu L, Wang M, et al. Effect of cranial irradiation on sperm concentration of adult survivors of childhood acute lymphoblastic leukemia: a report from the St. Jude Lifetime Cohort Studydagger. Hum Reprod. 2017; 32(6):1192-201.

21. Levine JM, Whitton JA, Ginsberg JP, et al. Nonsurgical premature menopause and reproductive implications in survivors of childhood cancer: a report from the Childhood Cancer Survivor Study. Cancer. 2018;124(5):1044-52.

22. Chemaitilly W, Li Z, Krasin MJ, et al. Premature ovarian insufficiency in childhood cancer survivors: a report from the St. Jude Lifetime Cohort. J Clin Endocrinol Metab. 2017;102(7):2242-50.

23. Chow EJ, Stratton KL, Leisenring WM, et al. Pregnancy after chemotherapy in male and female survivors of childhood cancer treated between 1970 and 1999: a report from the Childhood Cancer Survivor Study cohort. Lancet Oncol. 2016;17(5):567-76.

24. Green DM, Nolan VG, Goodman PJ, et al. The cyclophosphamide equivalent dose as an approach for quantifying alkylating agent exposure: a report from the Childhood Cancer Survivor Study. Pediatr Blood Cancer. 2014;61(1):53-67.

25. Bresters D, Emons JA, Nuri N, et al. Ovarian insufficiency and pubertal development after hematopoietic stem cell transplantation in childhood. Pediatr Blood Cancer. 2014; 61(11):2048-53.

26. Romerius P, Stahl O, Moell C, et al. High risk of azoospermia in men treated for childhood cancer. Int J Androl. 2011;34(1):69-76.

27. Green DM, Kawashima T, Stovall M, et al. Fertility of male survivors of childhood cancer: a report from the Childhood Cancer Survivor Study. J Clin Oncolz 2010;28(2):332-9.

28. Sklar CA, Mertens AC, Mitby P, et al. Premature menopause in survivors of childhood cancer: a report from the childhood cancer survivor study. J Natl Cancer Inst. 2006; 98(13):890-6.

29. Chemaitilly W, Mertens AC, Mitby P, et al. Acute ovarian failure in the childhood cancer survivor study. J Clin Endocrinol Metab. 2006;91(5):1723-8.

30. Meacham LRBK, Orwig K and Levine J. Standardizing risk assessment for treatment related gonadal insufficiency and infertility in childhood adolescent and young adult cancer: the PIN risk stratification system. J Adolesc Young Adult Oncol. 2020; In press.

31. George SA, Williamson Lewis R, Schirmer DA, et al. Early detection of ovarian dysfunction by anti-Mullerian hormone in adolescent and young adult-aged survivors of childhood cancer. J Adolesc Young Adult Oncol. 2019; 8(1):18-25.

32. Lie Fong S, Laven JS, Hakvoort-Cammel FG, et al. Assessment of ovarian reserve in adult childhood cancer 
survivors using anti-Mullerian hormone. Hum Reprod. 2009;24(4):982-90.

33. Lunsford AJ, Whelan $\mathrm{K}$, McCormick K, McLaren JF. Antimullerian hormone as a measure of reproductive function in female childhood cancer survivors. Fertil Steril. 2014;101(1):227-31.

34. Miyoshi Y, Ohta H, Namba N, et al. Low serum concentrations of anti-Mullerian hormone are common in $53 \mathrm{fe}$ male childhood cancer survivors. Hormone research in paediatrics. 2013;79(1):17-21.

35. van den Berg $\mathrm{MH}$, Overbeek A, Lambalk CB, et al. Longterm effects of childhood cancer treatment on hormonal and ultrasound markers of ovarian reserve. Hum Reprod. 2018; 33:1474-88.

36. Anazodo AC, Stern CJ, McLachlan RI, et al. A study protocol for the Australasian Oncofertility Registry: monitoring referral patterns and the uptake, quality, and complications of fertility preservation strategies in Australia and New Zealand. J Adolesc Young Adult Oncol. 2016; $5(3): 215-25$.

37. Liebenthron J, Montag M. Development of a nationwide network for ovarian tissue cryopreservation. In: Nagy ZP, Varghese AC, Agarwal A (Eds). Cryopreservation of mammalian gametes and embryos. New York: Humana Press; 2017; pp. 205-20.
38. Takai Y. Fertility preservation network in Asia: current status and issues of fertility preservation network in Japan. Onco Fertil J. 2018;1(2):71.

39. Geenen MM, Cardous-Ubbink MC, Kremer LC, et al. Medical assessment of adverse health outcomes in longterm survivors of childhood cancer. JAMA. 2007;297(24): 2705-15.

40. Bhakta N, Liu Q, Ness KK, et al. The cumulative burden of surviving childhood cancer: an initial report from the $\mathrm{St}$ Jude Lifetime Cohort Study (SJLIFE). Lancet. 2017; 390(10112):2569-82.

Address correspondence to:

Leena Nahata, MD

Center for Biobehavioral Health

Abigail Wexner Research Institute at Nationwide

Children's Hospital

$431 \mathrm{~S}$ 18th Street

Columbus, $\mathrm{OH} 43205$

USA

Email: leena.nahata@nationwidechildrens.org 